\title{
Un inusual bloom de Tetraselmis sp. en la Bahía de Valparaíso, Chile
}

\section{Unusual bloom of Tetraselmis sp. in the Valparaiso Bay, Chile}

\author{
Marlene Pizarro ${ }^{1}$, Rodolfo Iturriaga $^{2}$, Alejandro Silva $^{3} \&$ Sonia Gallegos $^{4}$ \\ ${ }^{1}$ Universidad de Valparaíso, Centro de Investigación y Gestión de los Recursos Naturales, Facultad de Ciencias, Gran Bretaña \\ 1111, Valparaíso, Chile. \\ 2University of Southern California, Department of Biological Sciences, Los Angeles, CA, 90089-0371, USA. \\ ${ }^{3}$ Universidad Católica de Valparaíso, Departamento de Oceanografía Física, Avenida Altamirano 1424, Valparaíso, Chile. \\ ${ }^{4}$ Naval Research Laboratory, Ocean Sciences, Stennis Space Center, MS, 39529, USA. \\ manepizarro@gmail.com
}

\begin{abstract}
A bloom of Tetraselmis sp. was observed during the first days of January of 2006 in the Valparaiso Bay $\left(32^{\circ} 57^{`} \mathrm{~S} ; 71^{\circ}\right.$ $\left.33^{\prime} \mathrm{W}\right)$, producing green coloration of sea and exclusion of phytoplankton species. Previous blooms of Tetraselmis sp. have not been observed in this bay.
\end{abstract}

La Bahía de Valparaíso durante primavera-verano se encuentra en una dinámica de surgencias, por lo que la población fitoplanctónica está dominada principalmente por diatomeas (Avaria 1971, Alveal \& Avaria 1982).

Tetraselmis spp. son fitoflagelados que pertenecen al grupo Prasinophyceae, registrado en la Bahía de Valparaíso (Padilla 1975). No es representativo del fitoplancton del área, sin embargo, una especie de este grupo produjo un bloom en toda la bahía.

Collantes \& Prado (2006) registraron una densidad entre 3 a $5 \times 10^{5} \mathrm{cel} \mathrm{ml}^{-1}$, produciendo una coloración verde en el mar y caracterizándose por ser monoespecífico, cambiando la composición poblacional del fitoplancton típico de surgencia durante ese periodo. Ocasionalmente se presentó escasa presencia de las especies Asteromonas gracilis, Chlamydomonas sp., Chaetoceros sp. y Skeletonema costatum. Las concentraciones de nitratos

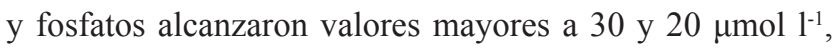
respectivamente. Sin embargo, el 5 de enero de 2006, los niveles de fosfato decrecieron abruptamente entre 0,3 y 0,8 $\mu$ mol $1^{-1}$ y al mismo tiempo los niveles de nitrato cayeron bajo el límite de detección.

Otros dos blooms de Tetraselmis sp. han sido descritos en diferentes lugares: uno en Frank Kitts Lagoon, Wellington Harbor, Nueva Zelanda el 10 de diciembre de 1993 (Jones \& Rhodes 1994) y el otro en Saldanha Bay, Sudáfrica, el 15 de enero de 2003 (Sym et al. 2004). Durante el bloom de Frank Kitts Lagoon, Tetraselmis sp. alcanzó concentraciones entre $773-1987,7 \times 10^{3}$ cel $1^{-1}$ y acompañados de otros fitoflagelados, como Cryptomonas spp. y Pyramimonas spp. que presentaron concentraciones entre $326-558 \times 10^{3}$ cel
$1^{-1}$. La temperatura superficial del mar se mantuvo entre 15 y $15,8{ }^{\circ} \mathrm{C}$ (Jones \& Rhodes 1994). Para el caso del bloom de la Bahía de Saldanha, ningún parámetro biológico ni ambiental fue medido (Sym et al. 2004). En ambos eventos se produjo la coloración verde.

Esta comunicación breve tiene por objetivo complementar el registro del bloom de Tetraselmis sp. ocurrido en la Bahía de Valparaíso, con mediciones de radiación ultravioleta $\mathrm{A}$ (UVA) y ultravioleta B (UVB) expresadas en anomalías, absorción espectral del material particulado $(A p(\lambda))$ y extinción de la luz, con el fin de describir las condiciones ambientales que se presentaron durante el bloom de enero de 2006.

Los datos de radiación ultravioleta (UV) fueron proporcionados por la Estación Campbell CR 10X, equipada con sensores NR-LITE-CNR1 a 60m sobre el nivel del mar de la Universidad Técnica Federico Santa María, Sede

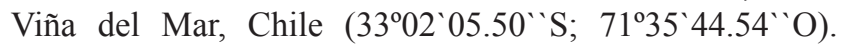
Los datos de magnitud - dirección del viento por la Gobernación Marítima de Valparaíso (3255`19.09'S, $\left.71^{\circ} 32^{`} 31.48^{`} \mathrm{O}\right)$. La absorción espectral del material particulado suspendido se estimó de acuerdo a Mitchell (1990), con un espectrofotómetro UV-VIS Shimadzu2500, en cuatro estaciones costeras a lo largo de la bahía (Estación 1, 32 ${ }^{\circ} 57^{`} 25,7^{`} \mathrm{~S}$; $71^{\circ} 33^{`}$ 08,2 `O, Estación 2, $32^{\circ}$ $59^{`} 54^{\prime} \mathrm{S} ; 71^{\circ} 33^{`} 06,4^{\prime} \mathrm{O}$, Estación $3,33^{\circ} 00^{`} 14,3^{\prime}{ }^{\prime} \mathrm{S} ; 71^{\circ}$ $33^{`} 10,6^{`} \mathrm{O}$, Estación $4,33^{\circ} 00^{`} 37,1^{\prime} \mathrm{S}$; $\left.71^{\circ} 33^{`} 22,6^{\prime} \mathrm{O}\right) \mathrm{y}$ las mediciones de atenuación de la luz fueron medidas con disco de Secchi.

Los niveles de radiación UV están expresados en anomalía diaria, tanto para UVA como para UVB. La data considerada 
para calcular esta anomalía fue desde el año 2002 al 2006. La anomalía es dada por la diferencia entre el valor máximo de radiación UVA y UVB diaria observadas desde diciembre 2005 a enero 2006 y el promedio de los valores de radiación UVA y UVB respectivamente, registrados a las $13 \mathrm{~h}$ desde el año 2002 al 2006. Una anomalía positiva indica que el nivel de radiación UV fue más alto que el registrado desde el año 2002 al 2006. Mientras que una anomalía negativa, indica lo contrario.

Las condiciones ambientales durante el bloom se caracterizaron por presentar los niveles más altos de radiación UVA y UVB registrados desde el año 2002 al 2006. Esto se puede observar en la anomalía positiva que produjo (Fig. 1) y baja magnitud de los vientos (Fig. 2c).
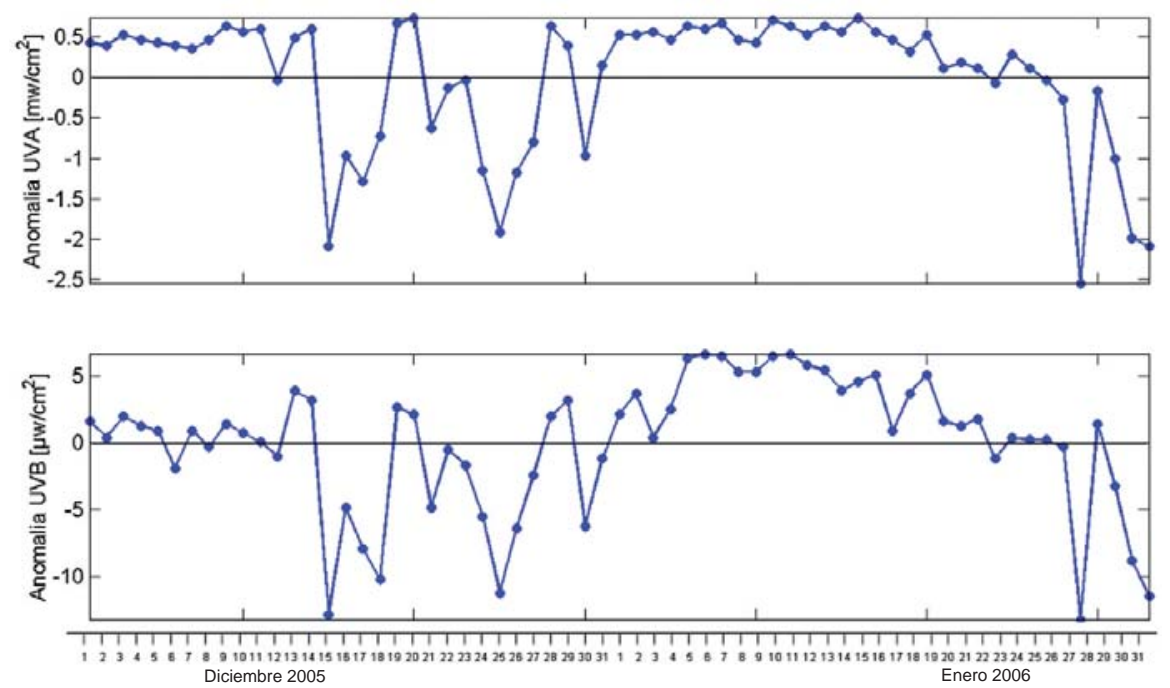

FigURA 1. Anomalía de la radiación UVA y UVB durante diciembre 2005 y enero 2006.

FIgURE 1. UVA and UVB radiation anomaly during December 2005 and January 2006.

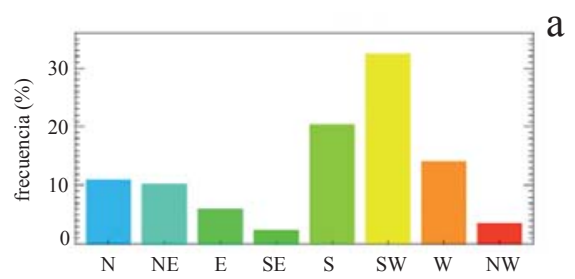

a

b
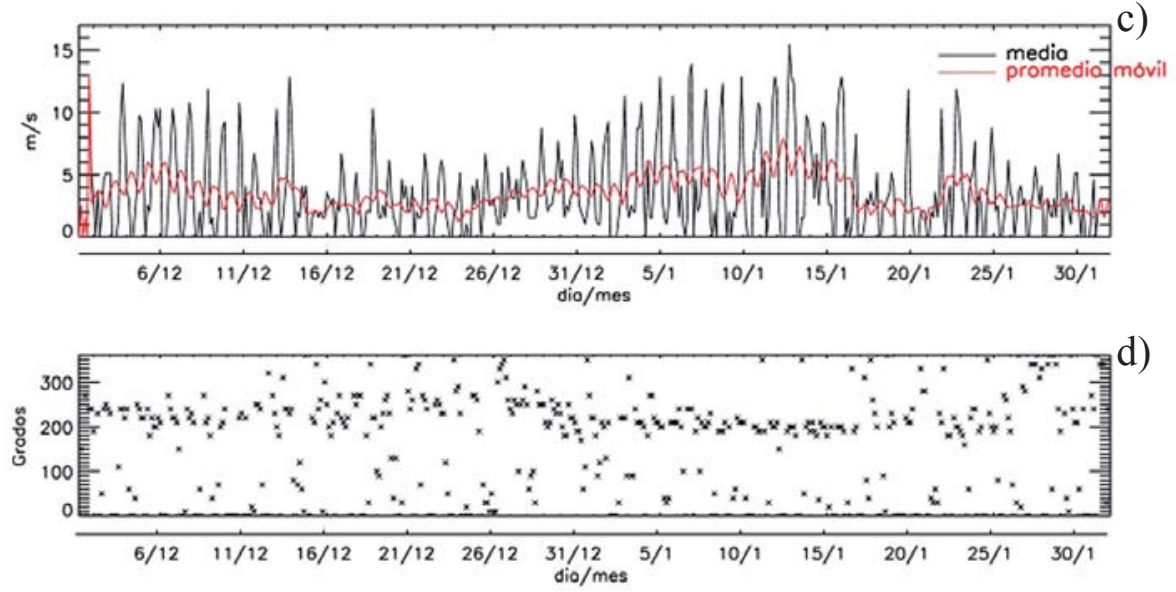

Figura 2. a) Gráfico de frecuencia (\%), b) Rosa de frecuencia de la dirección del viento, c) Magnitud del viento (m s${ }^{-1}$ ) y d) Dirección del viento (grados), durante diciembre 2005 y enero 2006.

Figure 2. a) Frecuency graphic (\%), b) Frecuency Rose of the direction of wind, c) Magnitude of wind ( $\mathrm{m} \mathrm{s}^{-1}$ ) and d) Direction of wind (degrees), during December 2005 and January 2006. 
Pizarro: Bloom de Tetraselmis en la Bahía de Valparaíso: Pizarro, M. ET AL.

Los gráficos de absorción espectral muestran peaks a $440 \mathrm{~nm}$ y $675 \mathrm{~nm}$, correspondiendo a la absorción de la clorofila $a$ y a $480 \mathrm{~nm}$ y $650 \mathrm{~nm}$ de la clorofila $b$. confirmando que el bloom estuvo formado específicamente por Tetraselmis sp. (Fig. 3) con elevadas concentraciones celulares, lo cual disminuyó la zona fótica a tres metros de profundidad (Fig. 4).
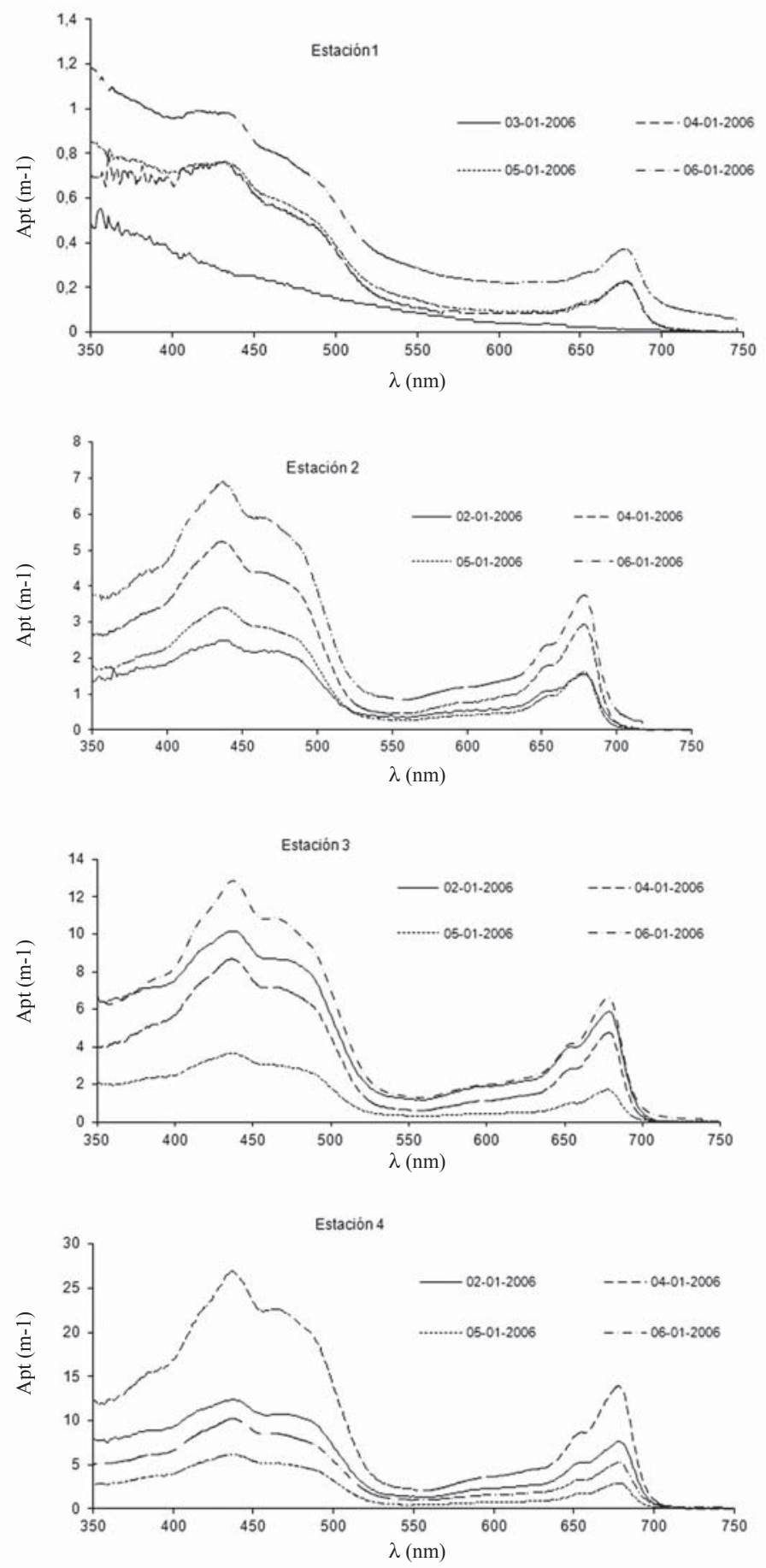

Figura 3. Absorción espectral del material particulado suspendido $\operatorname{Ap}(\lambda)$ de las cuatro estaciones de la Bahía de Valparaíso, durante los primeros días de enero de 2006.

FIGURE 3. Spectral absorption of suspended particulate matter $A p(\lambda)$, collected along the four stations of the Valparaiso Bay, during the first days of January of 2006. 
Cabe destacar la coincidencia temporal de los tres blooms de Tetraselmis sp.: durante diciembre y enero, un periodo correspondiente a los más altos niveles de radiación UV registrados en el Hemisferio Sur. Su alta tolerancia a la radiación UV está descrita (Jokiel \& York 1984, Montero et al. 2002).

En conclusión, el bloom de Tetraselmis sp. se desarrolló durante un periodo de altos niveles de radiación UV, baja magnitud de los vientos y elevadas concentraciones de nutrientes. La alta tolerancia a la radiación UV de Tetraselmis sp. pudo ser el factor excluyente de las especies de diatomeas, permitiendo alcanzar una alta densidad celular, debido al rápido consumo de nutrientes disponibles y en consecuencia reduciendo la zona fótica a sólo tres metros.

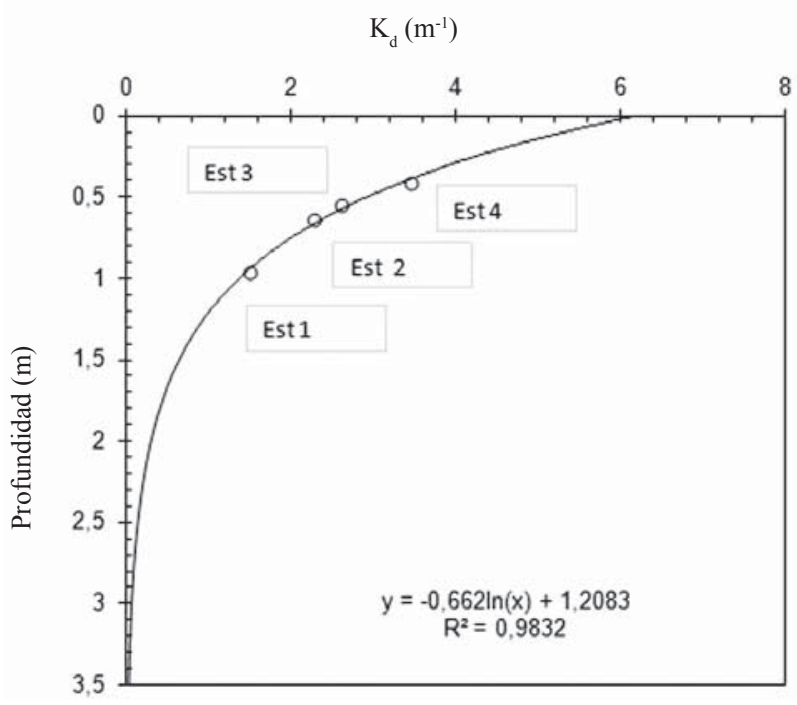

Figura 4. Coeficiente de atenuación de la luz $\mathrm{K}_{\mathrm{d}}\left(\mathrm{m}^{-1}\right)$ desde el 4 al 6 de enero de 2006.

Figure 4. Light attenuation coefficient $\mathrm{K}_{\mathrm{d}}\left(\mathrm{m}^{-1}\right)$ from 4 to 6 January 2006.

\section{AGRADECIMIENTOS}

A Gloria Collantes por identificación del género Tetraselmis. Universidad Técnica Federico Santa María. Gobernación Marítima de Valparaíso. Revisores por sus comentarios. Programa MECESUP-Chile.

\section{BIBLIOGRAFÍA}

Avaria, S. 1971. Variaciones mensuales del fitoplancton de la Bahía de Valparaíso entre Julio de 1963 y Julio 1966. Revista de Biología Marina y Oceanografía 14(3): 15-43.

Alveal, K. \& S. Avaria. 1982. Proliferación de primavera del fitoplancton en la Bahía de Valparaíso. II. Dinámica de las comunidades. Revista de Biología Marina y Oceanografía 18(1): $1-56$.

Collantes, G. \& R. Prado. 2006. Green bloom of Tetraselmis sp. in Valparaiso Bay. In: T. Wyatt (ed.), Harmfull Algae New, The Intergovernmental Oceanographic Commission, $\mathrm{N}^{\circ}$ 30, pp. 12. UNESCO, USA.

JOKIEL, P.L. \& R.H. YORK. 1984. Importance of ultraviolet radiation in photoinhibition of microalgal growth. Limnology and Oceanography 29(1): 192-199.

Jones, B. \& L. Rhodes. 1994. Suffocation of pilchards (Sardinops sagax) by a green microalgal bloom in Wellington Harbour, New Zealand. New Zealand Journal of Marine and Freshwater Research 28: 379-383.

Mitchell, B.G. 1990. Algorithms for determining the absorption coefficient of aquatic particulates using the quantitative filter technique (QFT). In: R.W. Spinrad (ed.), Ocean Optics X 1302: 137-148.

Montero, O., M. Klisch, D.P. Häder \& L. Lubian. 2002. Comparative sensitivity of seven marine microalgae to cumulative exposure to ultraviolet-B radiation with daily increasing doses. Botanica Marina 45: 305-315.

Padilla, M. 1975. Crecimiento poblacional de Tetraselmis suecica (Chlorophyceae) en ambiente controlado. Revista Biología Marina 15(3): 287-296.

Sym, S., F. Khosa, C. Marangoni \& G.C. Pitcher. 2004. On the identity of a bloom forming Tetraselmis species from Saldanha bay. 20th Congress of the Phycological Society of Southern Africa, Dissertation, University of the Witwatersrand, South Africa. 16 pp.

Recibido: 14.12.11

Aceptado: 17.04.12 